\title{
Rotational Stability of AcrySof Toric Intraocular Lens Over Time: Influence of Capsulorhexis Contraction
}

Joong Hee Kim

Kyong Jin Cho

Department of Ophthalmology, Dankook University College of Medicine, Cheonan, Korea

Received March 24, 2020

Accepted March 30, 2020

\footnotetext{
Correspondence

Kyong Jin Cho

Department of Ophthalmology, Dankook University Hospital, \#201 Manghyang-ro,

Dongnam-gu, Cheonan 31116, Korea

Tel.: +82-41-550-3945

Fax: +82-41-556-0524

E-mail: perfectcuredahanmail.net

(C) Korean Society for Laser Medicine and Surgery

(c) This is an open access article distributed under the terms of the Creative Commons Attribution NonCommercial License (http://creativecommons.org/ licenses/by-nc/4.0) which permits unrestricted noncommercial use, distribution, and reproduction in any medium, provided the original work is properly cited.
}

\author{
Background and Objectives \\ To evaluate the rotational stability of AcrySof toric intraocular lenses \\ (IOL) by considering lapse of postoperative time and influence of \\ capsulorhexis contraction.
}

\section{Materials and Methods}

A prospective, masked, single center study was conducted on 19 patients who had undergone microcoaxial cataract surgery and AcrySof toric IOL implantation. Slit-lamp retroillumination photographs of anterior segments were obtained from all patients after 1 week, 1 month and 3 months postsurgery. The degree of alteration of the postoperative IOL axis alignment and the amount of anterior capsular shrinkage were analyzed using Adobe Photoshop software.

\section{Results}

The mean degree of toric IOL axis misalignment was $2.18( \pm 20.2)$ degrees at 3 months follow-up. Quadrant analysis of the capsulorhexis aperture area at 1 week and 1 month post-operative, showed counterclockwise IOL rotation when the capsule contraction was dominant in the haptic part as well as clockwise rotation when dominant in the non-haptic part $(p=0.015)$.

\section{Conclusion}

The direction and degree of AcrySof toric IOL rotation differed throughout the follow-up period. Since most misalignments were found on the first post-operative day, physicians should try to minimize peri-operative risk factors that influence IOL rotation. There was also a correlation between the part of anterior capsule contraction and the direction of IOL rotation.

\section{Key words}

Capsulorhexis contraction; Rotational stability; Toric intraocular lens 


\section{INTRODUCTION}

Continued advances in techniques for intraocular lens (IOL) power calculations and measurements of axial lengths have led to more accurate IOL implantation during cataract surgery. Furthermore, improvements in surgical procedures, such as small-incision phacoemulsification and foldable IOL insertion, have minimized the occurrence of surgically induced astigmatism (SIA). These developments allow for better refractive correction, close to the targeted spherical equivalent. Cataract surgery is now regarded as a refractive surgery. However, many cataract patients have a significant degree of preexisting corneal astigmatism. In fact, it has been reported that $19 \%$ of cataract patients have a corneal astigmatism above 1.5 diopters (D) and $2 \%$ have astigmatism greater than $4 \mathrm{D}$. 'Despite the use of several surgical interventions for correcting astigmatism such as arcuate keratotomy, limbal relaxing incision, and laser in situ keratomileusis (LASIK), there are several drawbacks to these methods: risk of unintended corneal perforation, low predictability of remaining astigmatism, long-term refractive instability, and low time, effort, or cost effectiveness. ${ }^{2-7}$ In contrast, toric IOL implantation is known to be a safe, predictable, and simple technique for correcting astigmatism. ${ }^{8}$ Although early reports on toric IOL have demonstrated effective, immediate astigmatism correction, the subsequent appearance of IOL rotation has resulted in a reduction of this benefit. Indeed, it is known that a $30^{\circ}$ rotation of the IOL in the capsular bag after implantation can increase the astigmatism compared to the pre-operative value. $^{9-12}$

Recent studies have reported good rotational stability of AcrySof toric IOL (Alcon Laboratories, Inc., Fort Worth, TX, USA). ${ }^{13-16}$ However, the relationship of anterior capsule contraction and IOL rotation is not known. This paper seeks to evaluate the alignment of AcrySof toric IOL over time, as well as the relationship of anterior capsule contraction and IOL rotation.

\section{MATERIALS AND METHODS}

From March 2009 to December 2009, 19 eyes of consecutive 19 patients underwent microcoaxial cataract surgery and AcrySof toric IOL implantation by a single surgeon. Informed consent was obtained from all patients after explanation of possible surgical consequences and the need for an additional exam for the evaluation of rotational stability. Informed consent was obtained from all patients, and the study was performed according to the
Helsinki Declaration

Before surgery, all patients had a complete ophthalmic examination, including visual acuity (VA) (LogMAR), tonometry, autorefraction, slit lamp examination, fundoscopy, specular microscopy, and corneal topography (ORB scan II ${ }^{\circledR}$; Bausch and Lomb, Greenville, SC, USA). Patients with a senile cataract and a regular corneal astigmatism greater than $1.00 \mathrm{D}$ prior to surgery were included. Among these patients, those who had a corneal disorder affecting VA or corneal astigmatism, with history of glaucoma or retinal disorder, or with a previous history of intraocular or refractive surgery were excluded.

\section{Calculation of intraocular lens power}

The spherical IOL power and axial length were measured using the A-scan (Axis II ${ }^{\circledR}$; Quantel Medical, Clermont-Ferrand, France) and corneal astigmatism using the manual keratometry (DM- $4^{\circledR}$; Topcon Corp., Tokyo, Japan), respectively. Spherical IOL power was calculated using the SRK/T formula (A-constant 118.4). Cylindrical IOL power and axis were determined by a toric IOL calculator program lavailable at: http://www.acrysoftoriccalculator.com), which uses the patient's data, including the spherical IOL power, manual keratometry, location of incision and surgically induced astigmatism (SIA), to calculate the IOL power and axis.

\section{Reference markings and surgical technique}

Limbal reference markings were made using the toric reference corneal marker (AE-2793S, ASICO) with the patient seated upright on the slit-lamp and operating table right before the operation. The target axis was marked using a Mandez ring and a Nuijts toric axis marker (AE2740, ASICO) aligned with the pre-placed reference marks during the operation. One experienced surgeon performed all cataract operations under topical anesthesia. Continuous curvilinear capsulorhexis was performed under viscoelastic. Phacoemulsification was performed through a $2.2 \mathrm{~mm}$ temporal clear corneal incision using the Infinity Vision System (Alcon Laboratories, Inc.) and OZil Torsional Handpiece (Alcon Laboratories, Inc.). The toric IOL was implanted in the capsular bag using the Monarch injector system (Alcon Laboratories, Inc.). After irrigation and aspiration, the IOL axis was aligned with the limbal axis marks. The final incision size was $2.3 \mathrm{~mm}$ to 2.4 $\mathrm{mm}$ in all cases and no sutures were applied to the incision site. No intraocular complications, such as posterior capsule rupture, occurred. 


\section{Analysis of postoperative outcomes}

One week, one month and three months after surgery VA was measured and automated refractometer, slitlamp examination, and manual keratometry were performed. In all patients, the pupil was dilated to check the reference dots on the optic and slit-lamp retroillumination of the anterior segment was obtained using digital photographs at each follow-up visit. It was not possible to use any anatomical features to assist in checking that the horizontal was always in the same plane. However, because the camera position was fixed, and subject's head was relatively stable with the use of chin and head rests, it was felt there would not be significant deviation from the horizontal between images. The images were analyzed using the image-editing tools of Adobe Photoshop CS2 software. An independent observer determined the axis of IOL and the area of the capsulorhexis aperture as described below.

1. The axis of the IOL was determined using a theoretical line that connected the identifiable reference dots that were located on two opposite ends of the optic (Fig. 1A). The angle between this theoretical line and the horizontal was compared to the pre-operative, intended angle on the first day after operation. From the second follow-up appointment, the angle was compared with the value of the previously measured angle.

2. The size of the opening of the anterior capsule was determined using the Magnetic Lasso Tool (Adobe photoshop), and the number of pixels within the selected area was computed.

3. For standardization, the pixels of the area of the anterior capsule opening were divided by the pixels of an imagined circle, which was drawn based on the diameter determined by the distance of the two innermost reference dots on the optic (Fig. 1B). We tried to determine if this standardized value, which represents the amount of anterior capsule contraction, correlated with the degree of misalignment of the IOL axis.

4. For the evaluation of the relationship between the anterior capsule contraction and the direction of IOL rotation, we divided the anterior capsule opening area into four quadrants based on the IOL axis. The quadrant number was assigned based on the location of the haptics and optic of IOL (Fig. 2A, 2D). A standardized value was calculated for each quadrant. The change of this value and of the direction of the IOL rotation between one week and one month after operation were analyzed.

\section{Statistical analysis}

Statistical analysis was performed using SPSS for Windows, version 17.0 (SPSS Inc., Chicago, IL, USA). The numeric variables were summarized using the mean and standard deviation. Between-group comparisons were performed using the Wilcoxon signed-rank test. Friedman test was used to analyze the correlation between the rotation of intraocular lenses and the lapse of time after operation. All $p$-values were two-tailed, and a probability value of $p<0.05$ was considered statistically significant.

\section{RESULTS}

Nineteen eyes were included in this study. Eyes for which reference dots of the IOL optic could not be identified due to insufficient mydriasis or poor photo quality, were excluded from the analysis.

Among 19 eyes for which IOL rotation was identifiable at three months post-surgery, six eyes had underwent implantation with SN60T3, nine eyes with SN60T4, and four eyes with SN60T5. The amount of surgical induced astigmatism was 0.22 ( 0 to 0.75 ) D using the Cravy vector
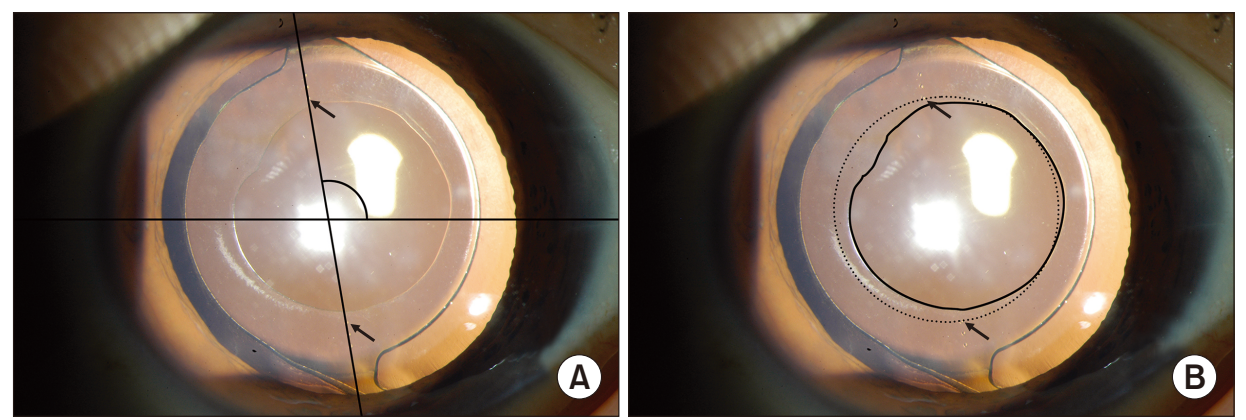

Fig. 1. Determination of the axis and anterior capsule opening area of IOL using (Black arrows: innermost reference dots on the optic surface). (A) IOL axis was determined by lining up opposite reference points on the optic surface. (B) Theoretical circle (dashed line) with its diameter as the distance between the innermost reference dots on the optic. Continuous curvilinear capsulorhexis (solid line, CCC) was computed using the Magnetic Lasso Tool. 

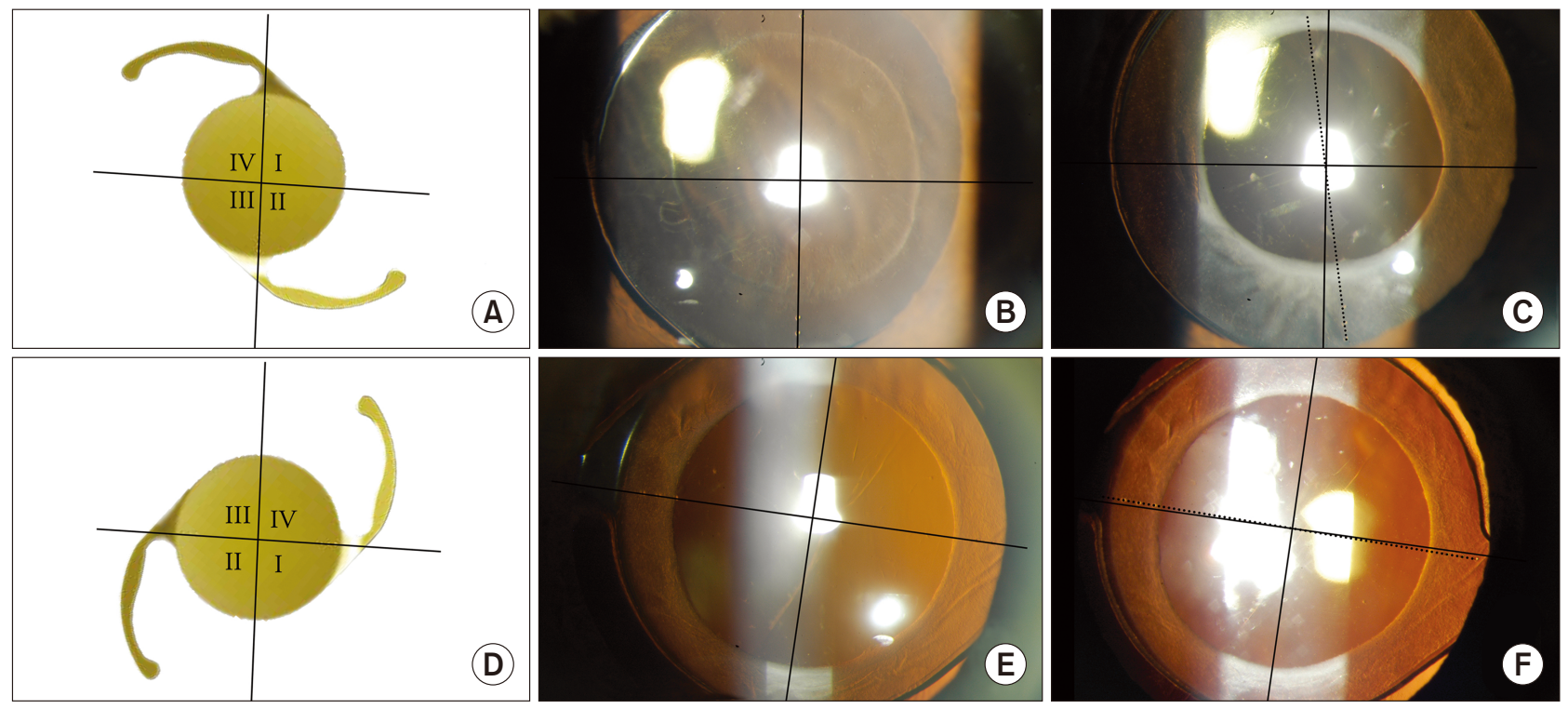

Fig. 2. The relationship between the change of the four quadrants of the anterior capsule opening area and the direction of IOL rotation. (A-C) Case 1, Eye with counterclockwise rotation. The Capsule contraction is dominant in quadrant II and IV (haptic part) resulting in the counterclockwise rotation of IOL $\left(90^{\circ}\right.$ to $95.1^{\circ}$ from one week to one month, postoperative). (D-F) Case 2. Eye with clockwise rotation. Capsule contraction is dominant in quadrant I and III (non-haptic part) resulting in clockwise rotation of IOL ( $-6.6^{\circ}$ to $-7.7^{\circ}$ from one week to one month, postoperative) (minus refers to counterclockwise direction from the target axis).

analysis. ${ }^{18}$ Although the mean corneal astigmatism was not statistically different after the operation compared to before the procedure, the mean refractive astigmatism and visual acuity improved significantly (Table 1). The mean degree of toric IOL axis misalignment was 2.18 I \pm 2.02) degrees at the three-month follow-up.

During follow-up, the number of eyes for which the direction and degree of IOL rotation could be determined and compared at one week, one month and three month after surgery was 16 (Table 2). The largest misalignment of the IOL axis was seen at the one day post-operative follow-up. The degree of misalignment decreased at each follow-up evaluation. Counterclockwise rotation was more frequent (10/16 eyes, $63 \%)$ at the first follow-up, while clockwise rotation was more frequent (11/16 eyes, $69 \%$ ) at one week post-surgery. The frequency of each rotational direction was nearly equal at the later follow-up evaluations.

The degree of axis rotation recorded between the first postoperative day and three months following surgery did not correlate with the area of the anterior capsule opening, measured at post-operative day one, nor with the difference in the area of the anterior capsule opening between one-day and three-months post-surgery $(r=0.273$ and 0.426 , respectively; $p=0.417$ and 0.399 , respectively). Between the one-week and one-month follow-up, sixteen eyes were used to perform the quadrant analysis of the
Table 1. Patient demographics, visual acuity, corneal and refractive astigmatism before and 3 months after operation

\begin{tabular}{lccc}
\hline \multicolumn{1}{c}{ Variables } & Preoperative & Postoperative & $p$-value * \\
\hline Eyes $(\mathrm{n})$ & \multicolumn{2}{c}{19} \\
Age (years) & \multicolumn{2}{c}{$62.21( \pm 13.13)$} & \\
UCVA (LogMAR) & $0.72( \pm 0.36)$ & $0.10( \pm 0.12)$ & $<0.001$ \\
BCVA (LogMAR) & $0.56( \pm 0.39)$ & $0.04( \pm 0.07)$ & $<0.001$ \\
Corneal astigmatism (D) & $1.86( \pm 0.73)$ & $1.86( \pm 0.86)$ & 0.871 \\
Refractive astigmatism (D) & $1.69( \pm 0.80)$ & $0.63( \pm 0.48)$ & 0.03 \\
\hline
\end{tabular}

*Wilcoxon signed ranks test.

area of the anterior capsule opening along with the IOL axis change. There was a tendency to counterclockwise rotation when the contraction was dominant in the haptic part (quadrant II or IV, Fig. 2) of the IOL and to a clockwise rotation when the contraction was dominant in the nonhaptic part (quadrant I or III, Fig. 2, Table 3) ( $p=0.015)$.

\section{DISCUSSION}

It is well known that AcrySof toric IOL implantation is an easy and effective tool for correcting corneal astigmatism, with good postoperative rotational stability. ${ }^{13-17}$ Recent studies of this novel IOL have reported that the mean degrees of its angle of rotation are from 3.35 to $3.63 .{ }^{13-15}$

The degree of IOL axis misalignment was largest one 
Table 2. Misalignment and direction of the AcrySof toric intraocular lenses rotation with the lapse of time after operation

\begin{tabular}{lcccc}
\hline & 7 days & 1 month & 3 months & $p$-value \\
\hline Degree of changes $(\mathrm{n}=16)$ & $1.13( \pm 1.71)$ & $1.92( \pm 2.01)$ & $2.18( \pm 2.02)$ & $<0.001$ \\
$\quad$ Direction and amount of misalignment (degrees) & $1.26( \pm 2.27)$ & $2.14( \pm 2.13)$ & $2.45( \pm 2.22)$ & 0.023 \\
$\quad$ Clockwise $(\mathrm{n}=8)$ & $0.99( \pm 1.05)$ & $1.70( \pm 2.00)$ & $1.91( \pm 1.91)$ & 0.007 \\
$\quad$ Counterclockwise $(\mathrm{n}=8)$ & &
\end{tabular}

*Friedman test.

Table 3. The relationship between the part of anterior capsule contraction and the direction of IOL rotation (between one week and one month follow up)

\begin{tabular}{lccc}
\hline \multirow{2}{*}{$\begin{array}{c}\text { Direction of IOL } \\
\text { rotation }\end{array}$} & \multicolumn{2}{c}{ Dominant part of capsule contraction } & \\
\cline { 2 - 3 } & Haptic & Non-haptic & \\
\hline Clockwise & 2 & 6 & 0.015 \\
Counterclockwise & 7 & 1 & \\
\hline
\end{tabular}

*Chi square test.

day post-surgery; this observation suggests that the cause of IOL rotation is strongly influenced by some perioperative factors. These factors can be classified into 2 categories: failure to achieve an accurate alignment along the intended axis under the microscope during surgery or immediate rotation of IOL, despite an accurate insertion of the IOL.

AcrySof Toric IOL is an open-loop, modified singlepiece, hydrophobic acrylic polymer material with Lshaped haptics. This IOL is designed to rotate clockwise during surgery, and adheres to the capsule after expansion. Thus, the surgeon first inserts the IOL at an axis that is minimally deviated, counterclockwise, from the intended axis. After removal of viscoelastic materials, the IOL is rotated, clockwise, to align with the intended axis as determined by the protocol. During this process, capsule adhesion of the IOL can occur before the final alignment and can result in a misaligned axis. Additionally, we experienced some error associated with the corneal reference marks. Marking color was diminished or, in some patients, spread during the operation, and this may also result in an inaccurate IOL insertion. One report found that a large mark size made it difficult to ensure accuracy, resulting in a misalignment of \pm 3 degrees of the intended axis for $95 \%$ of the markings. ${ }^{19}$ Additional novel tools or methods are needed for more accurate marking.

The additional rotations that were sequentially measured after the first postoperative day were relatively minimal. These delayed rotations seem to be affected by various factors such as the size of the capsular bag, which is associated with the axial length, and the thickness of the IOL, although there was no statistical correlation between IOL rotation and axial length or IOL power in this study. In addition to these factors, IOL tilting and decentration, which was not observed in the current study, or progressive contraction of the capsule, may contribute to the gradual rotation for several months after an operation. Subcapsular fibrosis can cause contraction, which can result in the compression of the supporting area of the inserted IOL.

Significant rotation was also detected between the first and the seventh day follow-up evaluations. During this post-operative period, the direction was mainly clockwise. The mechanisms promoting this directionality are unclear but it may be due to the properties of the IOL to rotate clockwise. Considering the amount of rotation, this seems to be affected by a dynamic factor such as shrinkage of the capsular bag. Haptic compression or expansion may induce a torque on the optic causing it to rotate independently of the haptic. ${ }^{20}$ Compression will cause clockwise rotation provided a moment is generated about the center of the IOL. Expansion would, in contrast, produce anticlockwise rotation. Although the capsular bag shrinkage was not seen between one- and seven-days post-surgery, as determined by slit-lamp examination, in this study, Strenn et al. ${ }^{21}$ reported that the capsular bag diameter was reduced between one-day and one-week post-surgery, as determined by measuring the capsular tension ring. Therefore, this suggests rapid shrinkage of the capsular bag following implantation. This can also be accompanied by IOL rotation.

Gradual capsular contraction can lead to a delayed IOL rotation because the capsule tissue is attached to the IOL. Although, the total anterior capsule opening size did not have a statistical correlation with the degree of IOL rotation in this study, we could predict that IOL rotation was attributed to the capsule contraction. Because the quadrant analysis of the area of the anterior capsule opening showed a comparatively constant result. There was a tendency to counterclockwise rotation when contraction was dominant in haptic part (quadrant II or IV) of IOL and 
clockwise rotation when dominant in the non-haptic part (quadrant I or III). The cause of this trend is uncertain but one hypothesis is that since the haptics have a shape that extends in the counterclockwise direction from the IOL optic, when compressed, the entire IOL can rotate to the same direction. A clinical study has shown that a reduction in circumference of the capsular bag is universal, implying that compression of the haptic is more likely than expansion. ${ }^{21}$ The counterclockwise rotation observed in our study is therefore likely to represent rotation of the haptic within the bag rather than that caused by haptic expansion. In contrast, when the compression is towards the center of the optic portion of IOL, the entire lens rotates clockwise due to the inherent properties of the IOL.

Several methods have been proposed to prevent continuous curvilinear capsulorhexis (CCC) contraction. Careful anterior capsular polishing and removal of the anterior subcapsular epithelial cells are important. An effective hydrodissection also helps to make it easier to remove the lens substance, ensuring a more complete removal of the cortex and associated cells. Large CCC (> $5.5 \mathrm{~mm}$ in diameter) may also be beneficial; small CCC have been found to correlate with lens capsular contraction. ${ }^{22,23}$ Although the degree of IOL rotation decreased over time, there were a number of eyes that displayed a small amount of IOL rotation up until the three-month follow-up evaluation. Thus, additional rotation may occur over a longer time period and further observation is needed.

The accuracy of measuring IOL rotation can be questioned because of possible errors arising from any head tilt during photography or imprecise framing of images. That is the main limitation of this study. However, to minimize this error and to achieve objectivity during the determination of the angle of IOL misalignment and the amount of capsulorhexis aperture area, anterior segment photographs were taken by one experienced examiner with a same magnifying power (10x). Magnifying power compensation of the computerized images was done by an independent observer using our specific standardization protocol which was mentioned above. The use of digital imaging to assess rotation has been shown to be an accurate, visual method of evaluating IOL axis alignment that may reduce the errors of measuring $I O L$ rotation with standard photography. ${ }^{24}$ Both this study and the previous smaller-scale assessment of AcrySof SA60AT used a similar methodology for assessing rotation digitally. ${ }^{25}$ Our results compare well with those in other studies of the same toric IOL design. The rotational stability of the AcrySof toric IOL in our study compares well with findings in
theU.S. FoodandDrugAdministration (FDA) AcrySof Toric IOL clinical trial. The mean rotation in the FDA study was less than 4.0 degrees.

In conclusion, we have shown that the direction and the degree of AcrySof toric IOL rotational changes during the post-operative period. The most severe misalignment was observed one day after surgery and care should be taken to minimize the peri-operative risk factors that influence this early IOL rotation. There was a tendency to counterclockwise rotation when contraction was dominant in haptic (closed loop) part of IOL and clockwise rotation when dominant in the non-haptic (open loop) part.

\section{ACKNOWLEDGEMENTS}

The English in this document has been checked by International Science Editing, editing company. For a certificate, see: www.internationalscienceediting.com

\section{CONFLICT OF INTEREST}

The authors have no conflict of interest to disclose.

\section{FUNDING}

The present research was conducted by the research fund of Dankook University in 2018.

\section{REFERENCES}

1. Hoffer KJ. Biometry of 7,500 cataractous eyes. Am J Ophthalmol 1980;90:360-8.

2. Colin J, Cochener B, Le Floch G. Excimer laser treatment of myopic astigmatism. A comparison of three ablation programs. Ophthalmology 1998;105:1182-8.

3. Dausch D, Klein R, Landesz M, Schröder E. Photorefractive keratectomy to correct astigmatism with myopia or hyperopia. J Cataract Refract Surg 1994;20 Suppl:252-7.

4. Salchow DJ, Zirm ME, Stieldorf C, Parisi A. Laser in situ keratomileusis for myopia and myopic astigmatism. J Cataract Refract Surg 1998;24:175-82.

5. Knorz MC, Wiesinger B, Liermann A, Seiberth V, Liesenhoff H. Laser in situ keratomileusis for moderate and high myopia and myopic astigmatism. Ophthalmology 1998;105:932-40.

6. Lee KM, Kim EC, Kim MS. Excimer laser refractive surgery to correct anisometropia due to residual astigmatism after cataract surgery. J Korean Ophthalmol Soc 2008;49:1589-96.

7. Niles C, Culp B, Teal P. Excimer laser photorefractive keratectomy using an erodible mask to treat myopic astigmatism. J Cataract Refract Surg 1996;22:436-40. 
8. Till JS, Yoder PR Jr, Wilcox TK, Spielman JL. Toric intraocular lens implantation: 100 consecutive cases. J Cataract Refract Surg 2002;28:295-301.

9. Shimizu K, Misawa A, Suzuki Y. Toric intraocular lenses: correcting astigmatism while controlling axis shift. J Cataract Refract Surg 1994;20:523-6.

10. Werblin TP. Do three-piece PMMA IOLs rotate after implantation in the capsular bag? J Refract Surg 1995;11:468-71.

11. Sanders DR, Grabow HB, Shepherd J. The toric IOL. In: Gills JP, Martin RG, Sanders DR, editors. Sutureless cataract surgery: an evolution toward minimally invasive technique. Thorofare: Slack Inc; 1992. p.183-97.

12. Gills JP, Martin RG, Thornton SP, Sanders DR. Surgical treatment of astigmatism. Thorofare: Slack Inc; 1994. p.159-64.

13. Chang DF. Comparative rotational stability of single-piece open-loop acrylic and plate-haptic silicone toric intraocular lenses. J Cataract Refract Surg 2008;34:1842-7.

14. Mendicute J, Irigoyen C, Aramberri J, Ondarra A, Montés-Micó R. Foldable toric intraocular lens for astigmatism correction in cataract patients. J Cataract Refract Surg 2008;34:601-7.

15. Bauer NJ, de Vries NE, Webers CA, Hendrikse F, Nuijts RM. Astigmatism management in cataract surgery with the AcrySof toric intraocular lens. J Cataract Refract Surg 2008;34:1483-8.

16. Rastogi A, Khanam S, Goel Y, Kamlesh, Thacker P, Kumar P. Comparative evaluation of rotational stability and visual outcome of toric intraocular lenses with and without a capsular tension ring. Indian J Ophthalmol 2018;66:411-5.

17. Zuberbuhler B, Signer T, Gale R, Haefliger E. Rotational stability of the AcrySof SA60TT toric intraocular lenses: a cohort study. BMC Ophthalmol 2008;8:8.

18. Cravy TV. Calculation of the change in corneal astigmatism following cataract extraction. Ophthalmic Surg 1979;10:38-49.
19. Hill W, Potvin R. Monte Carlo simulation of expected outcomes with the AcrySof toric intraocular lens. BMC Ophthalmol 2008;8:22

20. Pärssinen O, Räty J, Klemetti A, Lyyra AL, Timonen J. Compression forces of haptics of selected posterior chamber lenses. J Cataract Refract Surg 1997;23:1237-46.

21. Strenn K, Menapace R, Vass C. Capsular bag shrinkage after implantation of an open-loop silicone lens and a polylmethyl methacrylate) capsule tension ring. J Cataract Refract Surg 1997;23:1543-7.

22. Trivedi RH, Werner L, Pandey SK, Peng Q, Arthur S, Izak AM, et al. Intraocular lens-related opacifications: in front of, on, within, between, and behind the intraocular lens. In: Steinert RF, Fine $\mathrm{H}$, editors. Cataract surgery: techniques, complications and management. 2nd ed. St. Louis: Mosby; 2004. p.443-65.

23. Elmohamady MN, Elhabbak A, Gad EA. Circular YAG laser anterior capsulotomy for anterior capsule contraction syndrome. Int Ophthalmol 2019;39:2497-503.

24. Weinand F, Jung A, Stein A, Pfützner A, Becker R, Pavlovic S. Rotational stability of a single-piece hydrophobic acrylic intraocular lens: new method for high-precision rotation control. J Cataract Refract Surg 2007;33:800-3.

25. Kwartz J, Edwards K. Evaluation of the long-term rotational stability of single-piece, acrylic intraocular lenses. Br J Ophthalmol 2010;94:1003-6.

How to cite this article: Kim JH, Cho KJ. Rotational stability of AcrySof toric intraocular lens over time: influence of capsulorhexis contraction. Med Lasers 2020;9:44-50. https://doi.org/10.25289/ML.2020.9.1.44 